\title{
Functional Lipids as Nutraceuticals: A Review
}

\author{
Sneha S. Bhat \\ Guru Nanak Khalsa College of Arts, Science \& Commerce (Autonomous), Nathalal Parekh Marg, \\ Matunga East, Mumbai- 400019, Maharashtra, India
}

\begin{abstract}
Stephen L. Defelice, founder and chairman of the Foundation of Innovation Medicine, created the term "Nutraceuticals" in 1989 as a combination of the words "nutrition" and "pharmaceutical." Nutraceuticals are foodderived products that are claimed to give additional health advantages in addition to the fundamental nutritional content present in meals. Their kinds may be more significant than their quantity in terms of health and disease. The aim of this review is to provide a summary of the research on the role of functional lipids namely: Omega -6 fatty acid, Omega -3 fatty acid, Conjugated linoleic acid, Medium chain triglycerides and Phytosterols as nutraceuticals in human health. Functional lipids have been related to the prevention and treatment of a variety of ailments, according to new study. With the use of supplementary and dietary forms of functional lipids, scientific data has demonstrated positive improvements in patients and favorable benefits in healthy people.
\end{abstract}

Keywords: Nutraceuticals, Functional lipids, Conjugated linoleic acid, Medium chain triglycerides, Omega -6 fatty acid, Omega -3 fatty acid, Phytosterols

\section{INTRODUCTION}

A "nutraceutical" is a substance that is either a food or a component of food that delivers medical or health advantages, including illness prevention and treatment. Isolated nutrients, nutritional supplements, genetically modified "designer" foods, herbal products, and processed meals fall under this umbrella term, "Nutraceuticals". Nutraceuticals are difficult to distinguish from nutrients, food additives, medicines, by legal definition since they blur the boundary between food and pharmaceuticals.

According to the adage of a Greek physician Hippocrates (known as the founder of medicine), "let food be your medicine," the concept behind nutraceuticals is to focus on prevention. One of the most significant areas of research is its function in human nutrition, which has far-reaching significance for consumers, healthcare professionals, regulators, food producers, and distributors. The definition of nutraceuticals and associated products varies according to the source. They are classified based on their natural source, pharmacological characteristics, and chemical constitution.

Nutraceuticals have sparked great attention in recent years because of their potential nutritional, safety, and medicinal impacts. These compounds may have a role in a variety of biological activities, including antioxidant defenses, cell proliferation, gene expression, and mitochondrial integrity. As a result, nutraceuticals can be used to enhance health, prevent chronic illnesses, delay the aging process and therefore extend life expectancy, or simply to maintain the body's functions and integrity. These products are regarded as healthy sources for the prevention of life-threatening illnesses such as diabetes, renal and gastrointestinal problems, and other infections.

Aside from allergies, Alzheimer's disease, cardiovascular disease, cancer, eye problems, Parkinson's disease, and obesity, nutraceuticals have disease-modifying indications connected to oxidative stress. 


\section{FUNCTIONAL FOODS}

For a long time, it has been recognized that there is a definite link between the food we eat and our health. The present notion of functional foods arose from the growing understanding that consuming nutritious foods results in healthy diets, as well as the identification of the processes through which foods influence metabolism and health. In Japan, in the early 1980s, the phrase "functional food" was coined ${ }^{[1]}$. Error! Reference source not found.Although there is no unified definition of functional food, a common and basic definition is "processed foods that provide disease-prevention and/or healthpromoting advantages in addition to their nutritional value." Nutraceuticals, medicinal foods, and probiotic foods, all fall under the umbrella of functional foods. A diverse diet rich in fruits, vegetables, grains, legumes, and seeds provides the greatest health advantages. Fortified foods and dietary supplements, on the other hand, have been advertised, and the food industry has made functional food one of their current main trends ${ }^{[2]}$

Consumption of functional foods on a regular basis may be related with improved anti-oxidant, anti-inflammatory, insulin sensitivity, and anti-cholesterol effects.

The booming self-care movement, changes in food laws, and overwhelming scientific data showing the crucial relationship between nutrition and health have all contributed to a surge in interest in functional foods over the last decade ${ }^{[3]}$. Functional foods, in addition to novel food intended especially to improve health, can also include classic, familiar foods for which current study discoveries have highlighted new health advantages or debunked previous assumptions about potential detrimental health consequences.

The growth of scientific and public interest in nutrition's potential to prevent chronic illness and enhance health, which extends beyond the conventional focus on nutrient deficient illness prevention, has been critical to the growth of functional foods.

\section{FUNCTIONAL LIPIDS}

Lipids are a varied and ubiquitous collection of molecules that perform several important biological activities such as acting as structural components of cell membranes, storing energy, and engaging in signaling cascades $^{[4]}$. They are a prominent class of biological compounds that perform several important roles in many activities. Lipid diversity is on par with protein variety: cells express tens of thousands of distinct lipids and hundreds of proteins to control metabolism and transport ${ }^{[5]}$.

Fats and lipids are typical dietary components that may play important functions. Their kinds may be more significant than their quantity in terms of health and disease.

Although there is no actual definition of functional lipids, they can be described simply as a subset of functional foods that resemble traditional foods taken as part of a regular diet, they have been demonstrated to offer physiological advantages and/or lower the risk of chronic illness in addition to fundamental nutritional functions. ${ }^{[6]}$.

According to a new study, functional lipids have been related to the prevention and treatment of a variety of illnesses, including obesity, bone health, blood pressure, cardiovascular health, diabetes, treatment, and management of depression.

Omega-3 fatty acids that include alpha-linolenic acid (ALA), eicosapentaenoic acid (EPA) and docosahexaenoic acid (DHA), omega-6 fatty acids that include gamma linoleic acid, and linoleic acid, conjugated linoleic acid, medium-chain triglyceride oils, and phytosterols are examples of functional lipids.

\subsection{OMEGA-3 FATTY ACIDS}

Polyunsaturated fatty acids (PUFAs) containing more than one carbon-carbon double bond in their backbone are known as omega-3. 
Nutrition has the ability to have a significant impact on physical function and body metabolism. Particular emphasis has been put on omega-3 polyunsaturated fatty acids (n-3 PUFAs), which may be found in both terrestrial and marine environments.

N-3 PUFAs can help decrease inflammation and may lessen the risk of chronic illnesses including heart disease, cancer, and arthritis. They also control blood pressure, coagulation, glucose tolerance, nervous system growth, and function.

Omega-3 fatty acids are sometimes known as "vitamin F" from the word "fatty acids" "[8] and include ALA, EPA, and DHA.

Currently, marine fatty fish such as salmon, mullet, and mackerel are the primary sources of EPA and DHA for the human diet, and alternative EPA and DHA sources, such as bacteria, fungus, plants, and microalgae, are now being researched for commercial production ${ }^{[9]}$

ALA may be present in a variety of vegetables (spinach, broccoli, tomato, brussels sprouts, and rice bran) and meats in the form of lipoyllysine (ALA with binding lysine residues).

\subsubsection{OMEGA -3 FATTY ACIDS AS NUTRACEUTICAL \\ 3.1.2 Effect on Immune cells}

The immune system is a defensive mechanism that defends organisms against invading diseases such as viruses or bacteria. It is made up of a diverse array of cells, including immune cells as well as cellindependent processes. For decades, researchers have been studying the effects of dietary polyunsaturated fatty acids (PUFAs) on the immune system, with a particular focus on the omega-3 PUFAs. Omega-3 fatty acids have been shown to change three major aspects of macrophage biology: cytokine and chemokine synthesis and secretion, phagocytosis capability, and polarisation into conventionally activated or alternatively activated macrophages ${ }^{[12-13]}$.

Supplementation with omega-3 fatty acids has been found to be helpful in a variety of T-mediated illnesses, including autoimmune hepatitis ${ }^{[14]}$ and asthma ${ }^{[15]}$ Ex vivo, dietary supplementation with botanical omega-3 fatty acids reduces basophil production of leukotrienes in asthmatic patients ${ }^{[16]}$. Interestingly, dietary omega-3 fatty acids enhance particular immunological activities in certain immune cell types, such as phagocytosis by macrophages and neutrophils or Treg differentiation, suggesting that omega-3 fatty acids do not operate as unspecific immune-repressors ${ }^{[17]}$.

\subsubsection{Cardiovascular disease (CVD):}

In individuals with established coronary heart disease, omega-3 fatty acids have been found to dramatically lower the risk of sudden death due to cardiac arrhythmias ${ }^{[18]}$. Large-scale epidemiologic studies indicate that individuals at risk for coronary heart disease (CHD) benefit from eating omega-3 fatty acids from plants and seaweed. Although the optimum dosage is undefined, findings from prospective secondary prevention research suggest that EPA+DHA intakes ranging from 0.5 to 1.8 gramme per day (from fatty fish or supplements) are extremely advantageous $[19]$

A high EPA+DHA intake altered the expression of 1040 genes, whereas HOSF (high-oleic acid sunflower oil) consumption altered the expression of just 298 genes. EPA+DHA consumption reduced the expression of genes involved in inflammatory and atherogenic pathways, including nuclear transcription factor kappaB signalling, eicosanoid production, scavenger receptor function, adipogenesis, and hypoxia signalling ${ }^{[20]}$.There have been contradictory reports concerning EPA and DHA and their usage in severe coronary events following myocardial infarction. $\mathrm{EPA}+\mathrm{DHA}$ has been linked to a lower risk of recurrent coronary artery events and sudden cardiac death following an acute myocardial infarction percent as well as a lower risk of heart failure events ${ }^{[21-23]}$. 
Long-chain omega-3 fatty acids have an essential function in atherosclerosis stabilisation and lowering the risk of cardiovascular events. In fact, individuals with acute coronary syndrome had considerably lower blood levels of EPA and elongated metabolite of EPA and DPA than those without the condition ${ }^{[24]}$. A randomised experiment in type 2 diabetes patients revealed that supplementing with $1,800 \mathrm{mg}$ of EPA per day dramatically reduced CIMT(carotid intimal-medial thickness) and improved brachial-ankle pulse wave velocity, implying a reduction in atherosclerosis and enhanced endothelial function ${ }^{[25]}$.

Randomized controlled studies have conclusively shown that omega- 3 fatty acids can dramatically reduce the occurrence of CVD events in individuals with coronary artery disease. So far, the most compelling data comes from trials in which marinederived omega-3 fatty acids were taken as supplements or in fish. More clinical research is needed to establish ALA's cardioprotective effects. Although supplementations are a viable option, a food-based approach to boosting omega-3 fatty acids is preferable. More clinical and mechanistic research is needed to validate and clarify the health effects of omega-3 fatty acids for both primary and secondary prevention ${ }^{[19]}$.

\subsubsection{Alzheimer's disease (AD):}

$\mathrm{AD}$ is the most prevalent neurodegenerative disease in the elderly and the primary cause of dementia. DHA consumption is needed for proper neurodevelopment and brain health, especially during prenatal brain development ${ }^{[26]}$. Animal models of Alzheimer's disease show that a lack of DHA in brain tissue causes behavioural impairments, eventually leading to neurodegeneration and cognitive dysfunction similar to that seen in Alzheimer's patients ${ }^{[27-29]}$. Reduced DHA levels have been shown to have a negative impact on glutamate, a key excitatory neurotransmitter that contributes to the integrity of brain function in learning and memory performance ${ }^{[30]}$. DHA also appears to affect a variety of cellular activities, including increased membrane fluidity of amyloid precursor protein (APP) and a shift toward non-amyloidogenic processing, which inhibits and secretase, decreasing amyloid-release. [31] The findings of controlled trials done over the previous decade show that dietary supplementation with omega-3 fatty acids is only effective in the early stages of cognitive impairment. Epidemiological studies also show that a Mediterranean-style diet (high in plantderived ALA and Long chain-PUFA from fish and shellfish) may be protective against neurodegenerative disorders like Alzheimer's and Parkinson's ${ }^{[32]}$.

Consumption of long chain omega-3 PUFAs (omega-3 fatty acids) may delay cognitive decline and prevent the advancement of mental health diseases such as Alzheimer's disease, according to epidemiological and experimental research. Lower levels of omega-3 fatty acids in the erythrocyte membrane or plasma of individuals suffering from neurodegenerative disorders as compared to healthy persons demonstrate a link between mental health disorders and omega-3 fatty acids ${ }^{[33-35]}$.

\subsubsection{Foetal/Neonatal Development:}

Numerous advantages have been linked to the administration of omega-3 fatty acids throughout pregnancy and the postpartum period, whether through the diet or through supplements such as fish oil. Data from observational studies show that consuming omega-3 fatty acids during pregnancy, either through food or supplementation, is related with improved neurodevelopmental outcomes in the infant [36].

Children born to mothers who were identified with no seafood consumption had the highest risk of adverse or suboptimal outcomes, defined as testing in the lowest quartile for verbal and performance IQ at 
age 8 , behavioural problems at age 7 , and poor scores on early development tests evaluating fine motor skills, social skills, and communication skills as compared to those children born to mothers who consumed seafood during the pregnancy ${ }^{[37]}$

Omega-3 fatty acids appear to be essential during nursing as well. Fish oil supplementation during pregnancy and breastfeeding reduced the risk of new-born allergies. The overwhelming amount of evidence shows that omega-3 fatty acid consumption during pregnancy is critical for foetal brain growth and the child's future neurodevelopment, and that omega-3 fatty acid deficiency during pregnancy is related with poor developmental and behavioural scores.

\subsection{OMEGA-6 FATTY ACIDS}

Omega-6 fatty acids, like omega-3s, are polyunsaturated fatty acids, that may be found in vegetable oils, nuts, and seeds. The major dietary omega- 6 fatty acid is linoleic acid. Linoleic acid, when ingested, can be transformed to longer-chain fatty acids such as arachidonic acid by chain elongation and desaturation. In the body, omega-6 fatty acids have two primary roles: (1) they work as membrane structural components, modulating membrane function, and (2) they act as precursors of eicosanoids, which regulate renal and pulmonary function, vascular tone, and inflammatory responses [38]

\subsubsection{OMEGA- 6 FATTY ACIDS AS NUTRACEUTICAL:}

\subsubsection{Arachidonic Acid (ARA)}

Foods such as poultry, animal organs and meat, fish, seafood, and eggs can be the sources of Arachidonic acid. ARA is incorporated in phospholipids in the cytosol of the cell, which is adjacent to the endoplasmic reticulum membrane, that is densely packed with the proteins required for phospholipid synthesis and allocation to the various biological membranes ${ }^{[39]}$. As ARA is a basic component of cell structure, it will be especially important during development and growth, as well as in the event of severe or extensive cell damage and injury.

Polyunsaturated fatty acids (PUFAs), particularly ARA, influence the function of numerous ion channels, the activity of various enzymes, and are involved in cell apoptosis, necrosis, and death, all of which occur during embryogenesis and have a significant physiological and pharmacological impact on newborn health ${ }^{[40]}$ Children with autism exhibited lower blood PUFA levels, particularly ARA, than typical children, but showed significant improvement following dietary PUFA consumption ${ }^{\text {[41-42] }}$

PUFA, particularly free unesterified ARA, has been shown to have tumoricidal action in vitro and in vivo in previous studies. Human cervical cancer (HELA) cells and methyl cholanthrene-induced sarcoma cells were shown to be inhibited in vitro by free ARA. Free ARA increased the production of superoxide anion and lipid peroxidation in tumor cells, indicating a probable link between unesterified PUFA's capacity to enhance free radicals and their tumoricidal activity ${ }^{[43-44]}$.

ARA supplementation during resistance exercise may improve anaerobic capacity while reducing the inflammatory response to exercise. However, AA supplementation did not result in significantly higher increases in strength, muscle mass, or muscular hypertrophy indicators ${ }^{[45]}$. This fatty acid is mostly found in animal products such as chicken, beef, pork, and fish.

\subsubsection{Linoleic Acid (LA)}

Linoleic acid is the most often encountered PUFA in the human diet. Linoleic acid has four major fates after absorption. Like all fatty acids, it can be utilized as a source of energy. It may be esterified to generate neutral and polar lipids such phospholipids, triacylglycerols, and cholesterol esters. Linoleic acid, as a component of membrane phospholipids, functions as a structural component to maintain a level of membrane fluidity of the 
epidermis' transdermal water barrier. Furthermore, when liberated from membrane phospholipids, it can be enzymatically oxidized to a number of cell signaling derivatives ${ }^{[46]}$.

Overall, findings from clinical trials and meta-analyses show a link between high dietary intakes or tissue levels of n-6 PUFA, especially LA, and reduced cardiovascular risk (primarily of the plasma lipid profile), as well as improved long-term glycemic management and insulin resistance ${ }^{[48]}$.

\subsubsection{Conjugated Linoleic Acid (CLA)}

CLA, as minor lipids with a claimed functional dietary status, is gaining popularity in the treatment of major illnesses in humans such as cancer, atherosclerosis, and diabetes. CLA supplementation has also been utilized in sports in recent years, with the goal of reducing body fat and perhaps improving performance ${ }^{[47]}$ CLA was initially found as a fatty acid with significant anticarcinogenic characteristics, but research on its health impacts has expanded to include anti-obesogenic and anti-atherosclerotic qualities [49] Polyunsaturated fatty acids (PUFAs) like CLA and its metabolites can impact gene transcription at the nucleus level by interacting with other nuclear receptors and transcription factors ${ }^{[50]}$.

CLA may aid in decreased energy or food intake and increased energy expenditure, as well as decreased preadipocyte differentiation and proliferation, decreased lipogenesis and enhanced lipolysis and fat burning. CLA intake leads to fat and total body weight loss, lowers plasma concentrations of total and low density lipoprotein (LDL) cholesterol, and has an anti-inflammatory impact, according to animal and human studies.

\section{CLA suppresses cancer by} preventing tumor development and metastatic metastasis. It has a rapid onset of action and begins to suppress both malignant and benign tumors nearly immediately ${ }^{[52]}$. The 10-CLA isomer appears to act mainly through modulating apoptosis and cell cycle regulation, whereas the 9-CLA isomer influences arachidonic acid metabolism ${ }^{[53]}$.

Many studies strongly show that the 10-CLA isomer is the bioactive isomer of CLA that influences the bodyweight alterations seen in type II diabetes patients [54]. Another mechanism by which CLA may alleviate hyperinsulinemia (in Zucker diabetic fatty rats) is through the sensitization of adiponectin, a recently discovered hormone produced by adipocytes that have been shown to improve insulin sensitivity ${ }^{[55]}$.

CLA is a powerful anti-atherogenic dietary fatty acid that activates PPARs (Peroxisome proliferator-activated receptor) in animal models of atherosclerosis. Surprisingly, LDL cholesterol to HDL cholesterol ratios and total cholesterol to HDL cholesterol ratios were considerably lower in CLA-fed rabbits with less atherosclerosis ${ }^{[56-57]}$ Studies demonstrate that 9-CLA promotes the development of mineralized bone nodules using human bone cells and offer evidence for CLA isomerspecific effects on bone health ${ }^{[58]}$. CLA is mostly found in dairy products, although it is also present in ruminant meat.

\subsection{RATIO OF OMEGA-6/OMEGA-3}

Linoleic acid, an omega-6 fat, and the ARA it produces are crucial in reactions such as redness, swelling, heat, and discomfort. Acute inflammatory reactions, on the other hand, are intended to be swiftly reduced by resolvins derived from longchain omega-3 fatty acids, EPA and DHA. Thus, a healthy omega-6/3 ratio in the diet may be crucial for preventing an excessive and protracted inflammatory response, which might lead to tissue damage and, potentially an autoimmune disease.

A high omega-6/3 ratio predisposes to supraphysiologic inflammatory responses and contributes to persistent low-grade inflammation. Overconsumption of linoleic acid, mostly from synthetic omega- 6 seed oils, and a deficiency of EPA and DHA 
have been hypothesized to cause the population to become pro-inflammatory and pro-thrombotic ${ }^{\text {[59-60]. }}$.

In both animal and human research, a high omega- 6 fatty acid consumption and a high omega-6/omega-3 ratio are linked with weight growth, whereas a high omega3 fatty acid intake reduces the risk of weight gain. In animals, lowering the LA/ALA ratio reduces overweight and obesity.

Intake of plant oils high in n-6 PUFA and comparatively low consumption of marine foods (high in n-3 PUFA) raises the $n-6 / n-3$ ratio. When one consumes a diet high in ALA and low in LA, the levels of EPA and DHA in muscle tissue rise due to less competition for $\Delta 6$ desaturase. The n6/n-3 consumption ratio in most Indian consumers is $1 / 30-70$, whereas the optimum ratio is $1 / 5-10$ to preserve human health ${ }^{[84]}$.

It is critical to make every attempt to reduce omega-6 fatty acid intake while boosting omega-3 fatty acid intake. This could be implemented by (1) switching from omega-6 fatty acid-rich dietary vegetable oils (corn oil, sunflower, safflower, cottonseed, and soybean oils) to omega-3rich oils (flax, perilla, chia, rapeseed) and monounsaturated oils like olive oil, hazelnut oil, or the new high monounsaturated sunflower oil and (2) increasing fish consumption to 2-3 times per week while lowering meat consumption ${ }^{[61]}$.

\subsection{MEDIUM CHAIN TRIGLYCERIDES (MCT)}

Medium-chain triglyceride oil, as the name implies, contains medium-length chains of lipids known as triglycerides. MCTs are more easily absorbed than longerchain fatty acids present in many other meals due to their shorter length. During digestion, MCTs are hydrolyzed more quickly and thoroughly. The majority of the non-hydrolyzed MCTs that remain are easily absorbed by intestinal cells.

MCT oil is most typically derived from coconut oil, as MCTs account for more than half of the fat in coconut oil. These fats can also be present in foods like palm oil and dairy products ${ }^{[62]}$.

MCT has been used for decades to treat a variety of metabolic and digestive disorders, including pancreatic insufficiency, fat malabsorption, poor lymphatic chylomicron transfer, severe hyperchylomicronemia, and complete parenteral feeding. MCTs are also included in newborn formulae for premature babies ${ }^{[63]}$ MCT-supplemented diets are a potential strategy in the fight against adipogenic and steatogenic illnesses. Feeding healthy rats MCT-containing diets vs LCT (long chain triglycerides) containing diets significantly reduces fat deposition without altering whole-body protein content or absorption [64] The effect of MCT intake on body weight varies significantly depending on the study and diet regimen design. Women on a one-month diet with MCT supplying 30\% of total calories exhibited better fat oxidation and energy expenditure compared to women on LCT-containing meals, as well as a tendency to lose bodyweight ${ }^{[65]}$ An immediate decrease in food intake caused by MCT oil consumption is related to an increase in Leptin, a hormone with a wellknown anti-orexigenic action [66] Rising lactate levels during exercise may have a negative impact on performance. Surprisingly, MCTs may aid in the reduction of lactate accumulation. Previous research discovered that athletes who had 6 grams, or approximately 1.5 teaspoons, of MCTs with meal before cycling had lower lactate levels and felt it easier to exercise than those who consumed LCT ${ }^{[67]}$.

According to one in-vitro investigation, MCT capric acid, enhanced seizure control better than a commonly used anti-epileptic medication ${ }^{[68]}$.

An MCT ketogenic diet provides an additional energy source: ketones. This may improve the survival of brain cells. It also inhibits a brain receptor that promotes memory loss. While hereditary factors play a role, research shows that 20 to 70 grammes of supplementary MCTs including caprylic or capric acid may help with mild 
to severe Alzheimer's symptoms ${ }^{[69-70]}$. A previous in-vitro study found that coconut oil, which contains several MCTs, reduced Candida albicans growth by $25 \%$. This yeast is widespread and can cause thrush and other skin diseases. An in-vitro study also found that coconut oil inhibited the growth of Clostridium difficile, a disease-causing bacterium. The ability of coconut oil to inhibit yeast and bacterial development may be attributed to the caprylic, capric, and lauric acid found in MCTs ${ }^{\text {[71-72]. }}$

\subsection{PHYTOSTEROLS (PS)}

Phytosterols are cholesterol-like compounds present in all plant sources, with vegetable oils having the greatest amounts. Although plants offer a lot of PS, betasitosterol, campesterol, and stigmasterol are the most abundant and frequently consumed in higher-order plants ${ }^{[74]}$ Plant sterols are more hydrophobic than cholesterol and have a stronger affinity for fat-digesting micelles. As a result, they can displace intestinal cholesterol from the micelles, therefore decreasing intestinal cholesterol absorption [73]

PS and its saturated form: phytostanols, are both found in plant seeds and grains. Nuts such as almonds, cashews, cocoa seeds (unsweetened chocolate), and peanut butter are common dietary sources.
PS supplementation has been shown to decrease total cholesterol (TC) and LDLC (Low-density lipoprotein-cholesterol) according to a vast pool of work dating back to the early 1950s. Previously, the effectiveness of food-based vs capsule PS carriers was fiercely debated; however, recent clinical trials have shown that oral PS supplements (capsules and tablets) had a similar LDL-C reducing response to fortified meals ${ }^{[76-80]}$.

Non-esterified cholesterol is integrated into bile salt micelles under normal physiological circumstances for presentation to the brush border of enterocytes, where membrane-associated transport proteins promote absorption ${ }^{[81]}$. However, in the presence of PS, there is a reduction in cholesterol incorporation into micelles. It is commonly assumed that reduced cholesterol micellization is caused by PS's intrinsic hydrophobic structural characteristics, which compete with and displaces cholesterol, resulting in greater fecal loss ${ }^{[83]}$. The main drawback is that they can interfere with carotenoid absorption, however, this can be compensated either in the diet or by including these compounds in suitable carriers. Phytosterols have also been shown to have anticancer effects and to modulate the immune system ${ }^{[83]}$.

Table 1: Dietary sources of Functional Lipids ${ }^{[85]}$

\begin{tabular}{|l|l|}
\hline \multicolumn{1}{|c|}{ Functional Lipid } & \multicolumn{1}{c|}{ Dietary Sources } \\
\hline Omega-3 Fatty Acid & $\begin{array}{l}\text { Eicosapentaenoic acid and docosahexaenoic acid: Fatty fish such as mackerel, sardine, tuna and micro } \\
\text { algae. } \\
\text { Alpha linolenic acid: Dark green leafy vegetables, flax seed oil, chia seed oil, egg, meat, sea buckthorn, } \\
\text { hemp seed oil, canola oil, walnuts, hazelnuts }\end{array}$ \\
\hline Omega-6 Fatty Acid & $\begin{array}{l}\text { Linoleic acids: Vegetable oil, salad dressing, nuts } \\
\text { Gamma linolenic acid: Black currant oil, evening primrose oil, borage oil }\end{array}$ \\
\hline Conjugated linoleic Acid & $\begin{array}{l}\text { Milk, meats (kangaroo meat), grass fed ruminants, egg yolk, fish, fresh ground beef, butter fat, plain } \\
\text { yogurt, cultured buttermilk, custard style yogurt, cheddar cheese. }\end{array}$ \\
\hline Medium Chain Triglyceride & Animal fat, palm oil, coconut oil, cocoa butter. \\
\hline Phytosterols & $\begin{array}{l}\text { Wheat germ, corn oil, canola oil, almonds, brussels, sprouts, flaxseed, peanut butter, cauliflower, olive oil, } \\
\text { sesame seeds. }\end{array}$ \\
\hline
\end{tabular}

\section{CONCLUSION}

Functional lipids are dietary components that have the potential to influence human health by decreasing illness risk and increasing quality of life.
They have been shown to be effective, and they are readily available and cost-efficient to incorporate in the food chain/diet. Nonetheless, additional research is needed to properly examine and clarify the dosages, 
dietary intake, effectiveness, and processes of the numerous functional lipids described. More research is needed to better understand the creation of reliable disease biomarkers, clinical trials, and long-term consequences of these lipids in people.

\section{Acknowledgement: None}

\section{Conflict of Interest: None}

\section{Source of Funding: None}

\section{REFERENCES}

1. Error! Reference source not found.Arihara, K. (2014). Functional Foods. Encyclopedia Of Meat Sciences, 32$36 . \quad$ https://doi.org/10.1016/b978-0-12384731-7.00172-0.

2. Riezzo, G., Chiloiro, M., \& Russo, F. (2005). Functional foods: salient features and clinical applications. Current drug targets. Immune, endocrine and metabolic disorders, 5(3), 331-337. https://doi.org/ $10.2174 / 1568008054863790$.

3. Hasler, C. (2000). The Changing Face of Functional Foods. Journal of The American College Of Nutrition, 19(sup5), 499S-506S. https://doi.org/10.1080/07315724.2000.107 18972.

4. Fahy, E., Cotter, D., Sud, M., \& Subramaniam, S. (2011). Lipid classification, structures and tools. Biochimica et biophysica acta, 1811(11), 637-647. https://doi.org/10.1016/j.bbalip. 2011.06.009.

5. Muro, E., Atilla-Gokcumen, G. E., \& Eggert, U. S. (2014). Lipids in cell biology: how can we understand them better?.Molecular biology of the cell, 25(12), 1819-1823. https://doi.org/ 10.1091/mbc.E13-09-0516.

6. Moreau, R.A., 2011. An overview of functional lipids. In: 102nd AOCS Annual Meeting and Expo, Duke Energy Center, Cincinnati, Ohio, USA.

7. Rebecca Wall, R Paul Ross, Gerald F Fitzgerald, Catherine Stanton, Fatty acids from fish: the anti-inflammatory potential of long-chain omega-3 fatty acids, Nutrition Reviews, Volume 68, Issue 5, 1 May 2010, Pages280-289, https://doi.org/10.1111/j.1753-4887.2010. 00287.x.

8. DeFilippis, A., \& Sperling, L. (2006). Understanding omega-3's. American Heart Journal, 151(3), 564-570. https://doi.org/ 10.1016/j.ahj.2005.03.051.

9. Adarme-Vega, T.C., Lim, D.K.Y., Timmins, M. et al. Microalgal biofactories: a promising approach towards sustainable omega-3 fatty acid production. Microb Cell Fact 11, 96 (2012). https://doi.org/10.1186/ 1475-2859-11-96

10. Szelagg M., Mikulski D., Molski M. Quantum-chemical investigation of the structure and the antioxidant properties of $\alpha$ lipoic acid and its metabolites. J. Mol. Modeling. 2012;18:2907-2916. doi: 10.1007/s00894-011-1306-y.

11. Akiba S., Matsugo S., Packer L., Konishi T. Assay of protein-bound lipoic acid in tissues by a new enzymatic method. Anal. Biochem. 1998;258:299-304. doi: 10.1006/ abio.1998.2615.

12. Magrum L.J., Johnston P.V. Modulation of prostaglandin synthesis in rat peritoneal macrophages with omega-3 fatty acids. Lipids. 1983;18:514-521.doi:10.1007/ BF02535390.

13. Schroit A.J., Gallily R. Macrophage fatty acid composition and phagocytosis: effect of unsaturation on cellular phagocytic activity. Immunology. 1979;36:199-205.

14. Li Y.L., Tang Y., Wang S.J., Zhou J., Zhou J., Lu X., Bai X.C., Wang X.Y., Chen Z.L., Zuo D.M. Endogenous n-3 Polyunsaturated Fatty Acids Attenuate $\mathrm{T}$ Cell-Mediated Hepatitis via Autophagy Activation. Front. Immunol. 2016;7:350. doi: 10.3389/fimmu. 2016.00350.

15. Farjadian S., Moghtaderi M., Kalani M., Gholami T., Teshnizi S.H. Effects of omega-3 fatty acids on serum levels of Thelper cytokines in children with asthma. Cytokine. 2016;85:61-66. doi: 10.1016/j.cyto.2016.06.002.

16. Arm J.P., Boyce J.A., Wang L., Chhay H., Zahid M., Patil V., Govindarajulu U., Ivester P., Weaver K.L., Sergeant S., et al. Impact of botanical oils on polyunsaturated fatty acid metabolism and leukotriene generation in mild asthmatics. Lipids Health Dis. 2013;12:141. doi: 10.1186/1476-511X12-141.

17. Gutiérrez, S., Svahn, S. L., \& Johansson, M. E. (2019). Effects of Omega-3 Fatty Acids 
on Immune Cells. International journal of molecular sciences, 20(20), 5028. https://doi.org/10.3390/ijms20205028

18. Jain, A. P., Aggarwal, K. K., \& Zhang, P. Y. (2015). Omega-3 fatty acids and cardiovascular disease. European review for medical and pharmacological sciences, 19 (3), 441-445.

19. Kris-Etherton, P., Harris, W., \& Appel, L. (2003). Omega-3 Fatty Acids and Cardiovascular Disease. Arteriosclerosis, Thrombosis, And Vascular Biology, 23(2), 151-152. https://doi.org/10.1161/01.atv. 0000057393.97337.ae

20. Bouwens, M., van de Rest, O., Dellschaft, N., Bromhaar, M. G., de Groot, L. C., Geleijnse, J. M., Müller, M., \& Afman, L. A. (2009). Fish-oil supplementation induces antiinflammatory gene expression profiles in human blood mononuclear cells. The American journal of clinical nutrition, 90(2), 415-424. https://doi.org/ 10.3945/ajcn.2009.27680

21. Kris-Etherton PM, Harris WS, Appel LJ. Fish consumption, fish oil, omega-3 fatty acids, and cardiovascular disease. Circulation. 2002; 106:2747-57.

22. Tavazzi L, Maggioni AP, Marchioli R, Barlera S, Franzosi MG, Latini R, Lucci D, Nicolosi GL, Porcu M, Tognoni G. Effect of n-3 polyunsatu- rated fatty acids in patients with chronic heart failure (the GISSI-HF trial): a randomised, double-blind, placebocontrolled trial. Lancet. 2008;372:1223-30.

23. Marchioli R, Barzi F, Bomba E, Chieffo C, Di Gregorio D, Di Mascio R, Franzosi MG, Geraci E, Levantesi G, Maggioni AP, et al. Early protec- tion against sudden death by n-3 polyunsaturated fatty acids after myocardial infarction: time-course analysis of the results of the Gruppo Italiano per lo Studio della Sopravvivenza nell'Infarto Miocardico (GIS-SI)-Prevenzione. Circulation. 2002;105:1897-903.

24. Amano T, Matsubara T, Uetani T, et al. Impact of omega-3 polyunsaturated fatty acids on coronary plaque instability: an integrated backscatter intravascular ultrasound study. Atherosclerosis. 2011; 218:110-6.

25. Mita $\mathrm{T}$, Watada $\mathrm{H}$, Ogihara $\mathrm{T}$, et al. Eicosapentaenoic acid reduces the progression of carotid intima-media thickness in patients with type 2 diabetes. Atherosclerosis. 2007; 191:162-7.
26. Moreira J. D., Knorr L., Ganzella M., et al. Omega-3 fatty acids deprivation affects ontogeny of glutamatergic synapses in rats: relevance for behavior alterations. Neurochemistry International. 2010;56 (67) 753-759. doi: 10.1016/j.neuint.2010. 02.010 .

27. Lim G. P., Calon F., Morihara T., et al. A diet enriched with the omega-3 fatty acid docosahexaenoic acid reduces amyloid burden in an aged Alzheimer mouse model. Journal of Neuroscience. 2005;25 (12):3032-3040. doi:10.1523/ JNEUROSCI.4225-04.2005.

28. Fernandes J. S., Mori M. A., Ekuni R., Oliveira R. M. W., Milani H. Long-term treatment with fish oil prevents memory impairments but not hippocampal damage in rats subjected to transient, global cerebral ischemia. Nutrition Research. 2008;28(11): 798-808. doi:

29. Pomponi M., Pomponi M. DHA deficiency and Alzheimer's disease. Clinical Nutrition. 2008;27(1):p.170. doi: 10.1016/j.clnu.2007. 10.009 .

30. Su H.-M. Mechanisms of n-3 fatty acidmediated development and maintenance of learning memory performance. Journal of Nutritional Biochemistry. 2010;21(5):364373. doi: 10.1016/j.jnutbio.2009.11.003.

31. Grimm M. O. W., Kuchenbecker J., Grosgen S., et al. Docosahexaenoic acid reduces amyloid $\beta$ production via multiple pleiotropic mechanisms. The Journal of Biological Chemistry. 2011;286(16):1402814039. doi: 10.1074/jbc.m110.182329.

32. Sofi F., Abbate R., Gensini G. F., Casini A. Accruing evidence on benefits of adherence to the Mediterranean diet on health: an updated systematic review and metaanalysis. The American Journal of Clinical Nutrition. 2010;92(5):1189-1196. doi: 10.3945/ajcn.2010.29673.

33. Cherubini A., Andres-Lacueva C., Martin A., et al. Low plasma N-3 fatty acids and dementia in older persons: the InCHIANTI study. Journals of Gerontology-Series A Biological Sciences and Medical Sciences. 2007;62(10):1120-1126. doi: 10.1093/gerona/62.10.1120.

34. Chiu C.-C., Frangou S., Chang C.-J., et al. Associations between n-3 PUFA concentrations and cognitive function after recovery from late-life depression. American Journal of Clinical Nutrition. 
2012;95(2):420-427. doi: 10.3945/ajen.111. 015784.

35. Heude B., Ducimetière P., Berr C. Cognitive decline and fatty acid composition of erythrocyte membranes-the EVA Study. The American Journal of Clinical Nutrition. 2003;77(4):803-808.

36. Makrides M, Gibson RA, McPhee AJ, et al. DOMInO Investigative Team, authors. Effect of DHA supplementation during pregnancy on maternal depression and neurodevelopment of young children: a randomized controlled trial. JAMA. 2010; 304:1675-1683.

37. Hibbeln JR, Davis JM, Steer C, et al. Maternal seafood consumption in pregnancy and neurodevelopmental outcomes in childhood (ALSPAC study): an observational cohort study. Lancet. 2007;369:578-585.

38. Mori, T., \& Hodgson, J. (2013). Fatty acids: Health Effects of Omega-6 Polyunsaturated Fatty Acids. Encyclopedia Of Human Nutrition, 209-214. https://doi.org/10.1016/ b978-0-12-375083-9.00100-8

39. Vance J.E. Eukaryotic lipid-biosynthetic enzymes: the same but not the same. Trends Biochem Sci. 1998;23(11):423-428

40. Ordway R.W., Singer J.J., Walsh J.V., Jr. Direct regulation of ion channels by fatty acids. TrendsNeurosci. 1991;14(3):96-100.

41. Meguid N.A., Atta H.M., Gouda A.S., Khalil R.O. Role of polyunsaturated fatty acids in the management of Egyptian children with autism. Clin Biochem. 2008;41(13):1044-1048.

42. Yui K., Koshiba M., Nakamura S., Kobayashi Y. Effects of large doses of arachidonic acid added to docosahexaenoic acid on social impairment in individuals with autism spectrum disorders: a doubleblind, placebo-controlled, randomized trial. J Clin Psychopharmacol. 2012;32(2): 200-206.

43. Sagar P.S., Das U.N. Cytotoxic action of cis-unsaturated fatty acids on human cervical carcinoma (HeLa) cells in vitro. Prostaglandins Leukot Essent Fatty Acids. 1995;53(4):287-299.

44. Ramesh G., Das U.N. Effect of cisunsaturated fatty acids on Meth-A ascitic tumour cells in vitro and in vivo. Cancer Lett. 1998;123(2):207-214.

45. Roberts, M.D., Iosia, M., Kerksick, C.M. et al. Effects of arachidonic acid supplementation on training adaptations in resistance-trained males. $J$ Int Soc Sports Nutr 4, 21 (2007). https://doi.org/10.1186/ 1550-2783-4-21.

46. Whelan, J., \& Fritsche, K. (2013). Linoleic acid. Advances in nutrition (Bethesda, Md.), 4(3), 311-312. https://doi.org/10.3945/ an.113.003772

47. Barone R, Macaluso F, Catanese P, Marino Gammazza A, Rizzuto L, Marozzi P, et al. Endurance exercise and conjugated linoleic acid (CLA) supplementation up-regulate CYP17A1 and stimulate testosterone biosynthesis. PLoSOne. 2013;8:e79686. doi: 10.1371/journal.pone.0079686.

48. Marangoni, F., Agostoni, C., Borghi, C., Catapano, A., Cena, H., \& Ghiselli, A. et al. (2020). Dietary linoleic acid and human health: Focus on cardiovascular and cardiometabolic effects. Atherosclerosis, 292, 90-98. https://doi.org/10.1016/ j.atherosclerosis.2019.11.018

49. den Hartigh L. J. (2019). Conjugated Linoleic Acid Effects on Cancer, Obesity, and Atherosclerosis: A Review of PreClinical and Human Trials with Current Perspectives. Nutrients, 11(2),370. https://doi.org/10.3390/nu11020370

50. Sampath H, Ntambi JM. Polyunsaturated fatty acid regulation of genes of lipid metabolism. An Rev Nutr. 2005;25:317-340. doi:10.1146/annurev.nutr.25.051804.10197

51. Salas-Salvado J, Marquez-Sandoval F, Bullo M. Conjugated linoleic acid intake in humans: A systematic review focusing on its effect on body composition, glucose, and lipid metabolism. Crit Rev Food Sci Nutr. 2006;46:479-488. doi:10.1080/ 10408390600723953.

52. Belury MA, Moya-Camarena SY, Lu M, Shi L, Leesnitzer LM, Blanchard SG. Conjugated linoleic acid is an activator and ligand for peroxisome proliferator-activated receptor-gamma (PPAR) Nutr Res. 2002; 22:817-824. doi: 10.1016/S0271-5317(02) 00393-7.

53. Ochoa JJ, Farquharson AJ, Grant I, Moffat LE, Heys SD, Wahle KW. Conjugated linoleic acids (CLAs) decrease prostate cancer cell proliferation: different molecular mechanisms for cis-9, trans-11 and trans-10, cis-12isomers. Carcinogenesis. 2004;25: 1185-1191. doi: 10.1093/ carcin/ bgh116.

54. Belury MA, Mahon A, Banni S. The conjugated linoleic acid (CLA) isomer, 
t10c12-CLA, is inversely associated with changes in body weight and serum leptin in subjects with type 2 diabetes mellitus. $J$ Nutr. 2003;133:257S-60S.

55. Nagao K, Inoue N, Wang YM, Yanagita T. Conjugated linoleic acid enhances plasma adiponectin level and alleviates hyperinsulinemia and hypertension in Zucker diabetic fatty (fa/fa) rats. Biochem Biophys Res Commun. 2003;310:562-566. doi: 10.1016/j.bbrc.2003.09.044.

56. Toomey S, McMonagle J, Roche HM. Conjugated linoleic acid: a functional nutrient in the different pathophysiological components of the metabolic syndrome? [Functional foods] Curr Opin Clin Nutr Metab Care. 2006;9:740-747. doi: 10.1097/ 01.mco.0000247465.34037.05.

57. Brown JM, McIntosh MK. Conjugated linoleic acid in humans: Regulation of adiposity and insulin sensitivity. $J$ Nutr. 2003;133:3041-3046.

58. Platt I, Rao LG, El-Sohemy A. IsomerSpecific Effects of Conjugated Linoleic Acid on Mineralized Bone Nodule Formation from Human Osteoblast-Like Cells. Exp Biol Medic. 2007;232:246-252.

59. DiNicolantonio JJ, O'Keefe JH. Importance of maintaining a low omega-6/omega-3 ratio for reducing inflammation. Open Heart. 2018;5:e00946.

60. DiNicolantonio JJ, JOK Importance of maintaining a low omega-6/omega-3 ratio for reducing platelet aggregation, coagulation and thrombosis. Open Heart. 2019;6:e01011.

61. Simopoulos A. P. (2016). An Increase in the Omega-6/Omega-3 Fatty Acid Ratio Increases the Risk for Obesity. Nutrients, 8(3), 128. https://doi.org/10.3390/ nu8030128.

62. Bach A.C., Babayan V.K. Medium-chain triglycerides: An update. Am. J. Clin. Nutr. 1982; 36:950-962.

63. Marten B., Pfeuffer M., Schrezenmeir J. Medium-chain triglycerides. Int. Dairy J. 2006;16:1374-1382. doi: 10.1016/ j.idairyj.2006.06.015.

64. Ling P.R., Hamawy K.J., Moldawer L.L., Istfan N., Bistrian B.R., Blackburn G.L. Evaluation of the protein quality of diets containing medium-and long-chain triglyceride in healthy rats. J. Nutr. 1986; 116:343-349.
65. St-Onge M.P., Bourque C., Jones P.J., Ross R., Parsons W.E. Medium- versus longchain triglycerides for 27 days increases fat oxidation and energy expenditure without resulting in changes in body composition in overweight women. Int. J. Obes. Relat. Metab. Disord. 2003;27:95-102. doi: 10.1038/sj.ijo.0802169.

66. St-Onge M.P., Mayrsohn B., O'Keeffe M., Kissileff H.R., Choudhury A.R., Laferrere B. Impact of medium and long chain triglycerides consumption on appetite and food intake in overweight men. Eur. J. Clin. Nutr. 2014;68:1134-1140. doi: 10.1038/ ejen.2014.145.

67. Nosaka, N., Suzuki, Y., Nagatoishi, A., Kasai, M., Wu, J., \& Taguchi, M. (2009). Effect of ingestion of medium-chain triacylglycerols on moderate- and highintensity exercise in recreational athletes. Journal of nutritional science and vitaminology, 55(2),120-125.

https://doi.org/10.3177/jnsv.55.120

68. Chang, P., Terbach, N., Plant, N., Chen, P. E., Walker, M. C., \& Williams, R. S. (2013). Seizure control by ketogenic dietassociated medium chain fatty acids. Neuropharmacology, 69,105-114. https://doi.org/10.1016/j.neuropharm.2012.1 1.004

69. Augustin, K., Khabbush, A., Williams, S., Eaton, S., Orford, M., \& Cross, J. et al. (2018). Mechanisms of action for the medium-chain triglyceride ketogenic diet in neurological and metabolic disorders. The Lancet Neurology, 17(1),84-93. https:// doi.org/10.1016/s1474-4422 (17)30408-8

70. Cunnane, S., Courchesne-Loyer, A., StPierre, V., Vandenberghe, C., Pierotti, T., \& Fortier, M. et al. (2016). Can ketones compensate for deteriorating brain glucose uptake during aging? Implications for the risk and treatment of Alzheimer's disease. Annals Of The New York Academy Of Sciences, 1367(1), 12-20. https:// doi.org/10.1111/nyas.12999.

71. Ogbolu, D. O., Oni, A. A., Daini, O. A., \& Oloko, A. P. (2007). In vitro antimicrobial properties of coconut oil on Candida species in Ibadan, Nigeria. Journal of medicinal food, 10(2), 384-387. https://doi.org/ 10.1089/jmf.2006.1209.

72. Shilling, M., Matt, L., Rubin, E., Visitacion, M. P., Haller, N. A., Grey, S. F., \& Woolverton, C. J. (2013). Antimicrobial 
effects of virgin coconut oil and its mediumchain fatty acids on Clostridium difficile. Journal of medicinal food, 16(12), 10791085.https://doi.org/10.1089/jmf.2012.0303.

73. Plat J, Mensink RP. Effects of plant sterols and stanols on lipid metabolism and cardiovascular risk. Nutr Metab Cardiovasc Dis 2001;11:31-40.

74. Phillips KM, Ruggio DM, AshrafKhorassani M. Phytosterol Composition of Nuts and Seeds Commonly Consumed in the United States. Journal of Agricultural and Food Chemistry, 2005; 53: 9436-9445.

75. Peterson DW, Nichols CW Jr., Shneour EA. Some relationships among dietary sterols, plasma and liver cholesterol levels, and atherosclerosis in chicks. $J$ Nutr, 1952; 47: 57-65.

76. Maki KC, Lawless AL, Reeves MS, Dicklin MR, Jenks BH, Shneyvas E, Brooks JR. Lipid-altering effects of a dietary supplement tablet containing free plant sterols and stanols in men and women with primary hypercholesterolaemia: a randomized, placebo-controlled crossover trial. Int J Food Sci Nutr, 2012; 63: 476-82.

77. Maki KC, Lawless AL, Reeves MS, Kelley KM, Dicklin MR, Jenks BH, Shneyvas E, Brooks JR. Lipid effects of a dietary supplement softgel capsule containing plant sterols/stanols in primary hypercholesterolemia. Nutrition, 2013; 29: 96-100.

78. Padro T, Vilahur G, Sanchez-Hernandez J, Hernandez M, Antonijoan RM, Perez A, Badimon L. Lipidomic changes of LDL in overweight and moderately hypercholesterolemic subjects taking phytosterol-and omega-3-supplemented milk. J Lipid Res, 2015; 56: 1043-56.

79. Ras RT, Fuchs D, Koppenol WP, Garczarek U, Greyling A, Keicher C, Verhoeven C, Bouzamondo $\mathrm{H}$, Wagner $\mathrm{F}$, Trautwein EA. The effect of a low-fat spread with added plant sterols on vascular function markers: results of the Investigating Vascular Function Effects of Plant Sterols
(INVEST) study. Am J Clin Nutr, 2015; 101: 733-41.

80. Rideout TC, Chan YM, Harding SV, Jones PJ. Low and moderate-fat plant sterol fortified soymilk in modulation of plasma lipids and cholesterol kinetics in subjects with normal to high cholesterol concentrations: report on two randomized crossover studies. Lipids Health Dis, 2009; 8: 45.

81. Amiot MJ, Knol D, Cardinault N, Nowicki M, Bott R, Antona C, Borel P, Bernard JP, Duchateau G, Lairon D. Phytosterol ester processing in the small intestine: impact on cholesterol availability for absorption and chylomicron cholesterol incorporation in healthy humans. J Lipid Res, 2011; 52: 1256-64.

82. Gleize B, Nowicki M, Daval C, Koutnikova H, Borel P. Form of phytosterols and food matrix in which they are incorporated modulate their incorporation into mixed micelles and impact cholesterol micellization. Mol Nutr Food Res, 2016; 60: 749-59.

83. Qúllez, J. (2003). Potential uses and benefits of phytosterols in diet: present situation and future directions. Clinical Nutrition, 22(4), 343-351. https://doi.org/ 10.1016/s02615614(03)00060-8.

84. Abedi, E., \& Sahari, M. A. (2014). Longchain polyunsaturated fatty acid sources and evaluation of their nutritional and functional properties. Food science \& nutrition, 2(5), 443-463. https://doi.org/10.1002/fsn3.121.

85. Alabdulkarim, B., Bakeet, Z., \& Arzoo, S. (2012). Role of some functional lipids in preventing diseases and promoting health. Journal of King Saud UniversityScience, 24(4),319-329.

https://doi.org/10.1016/j.jksus.2012.03.001.

How to cite this article: Bhat SS. Functional lipids as nutraceuticals: a review. International Journal of Science \& Healthcare Research. 2021; 6(4): 111-123. DOI: https://doi.org/ 10.52403/ijshr.20211018 